\title{
BMJ Open Investigating the impact of patient- centred labels on comprehension of medication dosing: a randomised controlled trial
}

\author{
Ahsan Saleem (D) , ${ }^{1,2}$ Gemma Woodruff, ${ }^{3}$ Kathryn Steadman, ${ }^{1}$ Adam La Caze ${ }^{1}$
}

To cite: Saleem A, Woodruff G, Steadman K, et al. Investigating the impact of patient-centred labels on comprehension of medication dosing: a randomised controlled trial. BMJ Open 2021;11:e053969. doi:10.1136/ bmjopen-2021-053969

- Prepublication history and additional supplemental material for this paper are available online. To view these files, please visit the journal online (http://dx.doi.org/10.1136/ bmjopen-2021-053969).

Received 29 May 2021 Accepted 29 0ctober 2021

Check for updates

(C) Author(s) (or their employer(s)) 2021. Re-use permitted under CC BY-NC. No commercial re-use. See rights and permissions. Published by BMJ.

${ }^{1}$ School of Pharmacy, The University of Queensland, Woolloongabba, Queensland, Australia

${ }^{2}$ Faculty of Nursing, University of Alberta, Edmonton, Alberta, Canada

${ }^{3}$ Pharmacy Department, Royal Brisbane and Women's Hospital, Herston, Queensland, Australia

Correspondence to

Dr Ahsan Saleem;

a.saleem@uq.edu.au

\section{ABSTRACT}

Objective This study aims to implement a version of patientcentred labels (PCL) consistent with current labelling practice in Australia; assess the effectiveness of PCL in relation to the proportion of participants that correctly comprehend dosing instructions, and explore the proportion of correct comprehension of PCL in participants with both low and high health literacy.

Design Randomised controlled trial.

Setting A large tertiary care hospital in Brisbane, Queensland, Australia.

Participants 121 participants with a majority born in Australia $(65.3 \%)$, New Zealand (14.0\%), the UK (6.6\%) and Ireland $(2.5 \%)$.

Intervention Participants were randomly assigned to either a panel of three PCL $(n=61)$ or three standard labels $(n=60)$ and asked to comprehend their assigned panel of labels.

Outcome measures Difference in the proportion of participants that correctly comprehend dosing instructions provided on PCL compared with standard labels. The twoproportion test was used to measure the impact of PCL on the proportion of participants correctly comprehending dosing instructions.

Results A greater proportion of participants were able to accurately comprehend PCL compared with standard labels. The proportion of participants who were able to correctly comprehend dose instructions provided on all three labels was significantly higher in the group that received PCL; $23.3 \%$ standard vs $83.6 \% \mathrm{PCL}, \mathrm{p}<0.001$. The effect was observed in both low and high health literacy participants. The proportion of participants with accurate label comprehension was higher in participants with low Newest Vital Signs scores $(8.3 \%$ standard vs $85.7 \%$ PCL, $p<0.001)$ and low Rapid Estimate of Adult Literacy in Medicine scores (10.5\% standard vs $96.0 \%$ PCL, $p<0.001$ ) who received PCL.

Conclusion This study supports the use of PCL in Australian pharmacy practice. PCL provide simple, clear and explicit dosing instructions to patients. Implementing PCL may reduce the risk of misinterpreting dosing instructions by patients and improve quality use of medicines.

Trial registration number ACTRN12621000083897; Results.

\section{INTRODUCTION}

Dispensing labels provide important instructions to patients about the appropriate use
Strengths and limitations of this study

- This randomised controlled trial (RCT) used a power sampling formula to calculate sample size, which enhances the generalisability of this RCT.

- Participants were randomly assigned to either patient-centred label or standard labels group using 1:1 simple randomisation technique.

- A limitation of this RCT is that it did not measure the actual medication adherence or medication-taking behaviour of participants.

- Findings of this RCT are not generalisable to minority groups as very few culturally and linguistically diverse participants were recruited.

of medicines. ${ }^{1}$ However, many patients face difficulty in interpreting dosage instructions, which may lead to medication misuse and adverse outcomes. ${ }^{2-4}$ Patients from both non-English speaking (culturally and linguistically diverse; CALD) and English speaking (non-CALD) backgrounds are at risk of misinterpreting dosage instructions, ${ }^{35}$ with approximately every one in two patients misinterpreting at least one label instruction. ${ }^{6}$ Several factors have been associated with misinterpreting dosage instructions, including low health literacy, cultural and linguistic barriers, and complexity of format and readability of dispensing labels. ${ }^{578}$

The extent to which patients can accurately interpret and understand dosage instructions relies on their ability to access, read and understand health information, which is referred to as 'health literacy'. ${ }^{3}$ Health literacy requires individuals to have specific sets of skills to adequately perform tasks in a variety of health-related situations. ${ }^{9}$ To accurately interpret and follow dosage instructions provided on a dispensing label, patients are required to have at least the three health literacy skills of word recognition and pronunciation, numeracy and comprehension. ${ }^{10}$ 
The content and format of the dispensing labels also play an important role in determining how well patients comprehend dosing instructions. The content and format of dispensing labels provided by pharmacies varies between and within jurisdictions. ${ }^{11-13}$ To standardise the content and format of dispensing labels and to improve the comprehension of dosage instructions by patients, the concept of patient-centred labels (PCL) has been introduced. ${ }^{1415} \mathrm{PCL}$ are designed to provide information in a more reliable way than standard labels. The available guidelines surrounding PCL ${ }^{1415}$ suggests that it should contain:

- Instructions in large font sizes (12 points or above).

- Information in lists rather than sentences.

- Dosage instructions in numbers rather than words (2 instead of TWO).

- More explicit instructions about drug use ('Take 1 tablet in the morning' instead of 'Take ONE tablet ONCE daily').

- White space and typographical cues (bolding and highlighting) to communicate important information.

- Information in Universal Medication Schedule (UMS) format to provide explicit information about dosing intervals to patients (morning, evening, etc).

Many studies from North America and Europe have reported that the implementation of PCL improve comprehension of dispensing label instructions in patients and reduce the risk of medication misuse. ${ }^{15-18}$ However, there has been limited work on implementing PCL in an Australian context and assessing whether PCL improve comprehension of key directions.

The sizing and standard formatting of dispensing labels in Australia are different to North America in which most studies assessing PCL have been conducted. There is no fixed standard label size in Australia and there are differences between states and territories as well as between community and hospital pharmacies. The size of dispensing labels used by many pharmacies in Australia is $80 \times 40 \mathrm{~mm},{ }^{19}$ but the actual white space for dosing instructions is much smaller because of stylistic margins or headers and footers pre-printed on labels. ${ }^{20}$ The Therapeutic Goods Administration advises a minimum dedicated space of $70 \times 30 \mathrm{~mm}$ for the dispensing label on medicine packaging, ${ }^{21}$ and manufacturers are required to adhere to this minimum size or leave a space as large as possible for dispensing label. The information provided on the dispensing label generally includes the name of the patient and prescribing health practitioner, medicine brand name, active ingredient and strength, dosing instructions, the pharmacist's details and other information relating to the prescription (see standard labels in online supplemental materials). ${ }^{21}$ The Australian Commission on Safety and Quality in Health Care (ACSQHC) organised a national round table conference in 2013 and made recommendations to optimise the quality of dispensing labels. ${ }^{22}$ The ACSQHC has recently finalised and released dispensing label standards. ${ }^{19}$ Most of the new labelling standards are in line with available guidance surrounding PCL with some new additions including signposting active ingredient/brand name and adding machine-readable verification code to be used during dispensing process among various others. ${ }^{19}$ While the supporting strategy 8 of the new labelling standards recommends keeping the size of the label to a minimum of $80 \times 40 \mathrm{~mm}$, increasing the size of the label to $90 \times 65 \mathrm{~mm}$ has been acknowledged to provide adequate space to allow instructions to be written in larger fonts, a key component of PCL.

Some preliminary work conducted by other groups in Australia has tested different formats and sizes of dispensing labels and reported that optimised dispensing labels written in numeric figures are better comprehended by participants. ${ }^{23-25}$ In one of those studies, no explicit information was provided on the label size or font size used in their study, ${ }^{24}$ and the other study kept font size equal or below 10 points for all small and large labels that they developed. ${ }^{25}$ To date, no randomised controlled trials have been conducted and reported to investigate the impact of PCL in Australia.

Research exploring the effectiveness of PCL may help pharmacy services administrators, regulatory authorities and policy-makers in developing effective strategies and policies to optimise the format and contents of dispensing labels and to improve the quality use of medicines. This study aims to implement a version of PCL that is consistent with current labelling practices but with large font size (12 points) and more white spacing for dosing instructions; assess the effectiveness of PCL in terms of the proportion of participants that correctly comprehend dosing instructions, and explore the proportion of correct comprehension of PCL in participants with both low and high health literacy.

\section{METHODS}

\section{Study design, participants and recruitment}

Recruitment took place between January and March 2020. Participants waiting for prescription filling or a medical appointment were recruited from the waiting area of pharmacy department in a 929-bed hospital (Royal Brisbane and Women's Hospital) located within Queensland's largest public health services provider (Metro North Hospital and Health Service) in Brisbane, Australia. Participants of all genders, aged 18 years or above, and either from English speaking background or non-English speaking background were invited to participate. No other criteria were used to exclude participants. We intended to approach, invite and recruit all participants from the waiting area of the hospital considering them to be representative sample of the general population. Two approaches were used to recruit participants: (1) pharmacy staff at the counter directed participants towards the researcher and explained the study purpose and (2) the researcher approached individuals directly, introduced himself and discussed study objectives. Participants were randomly assigned to either the control group 
(standard label) or intervention group (PCL) using 1:1 simple randomisation. A random sequence was generated on a paper before starting participant recruitment to avoid selection bias by the interviewer (AS).

Wolf et $a l^{16}$ showed that PCL increased the proportion of participants accurately interpreting dispensing labels from $69 \%$ to $91 \%$ when compared with standard labels (22\% increase in proportion). Based on this study, a minimum sample size of 102 participants (51 in each group) was calculated using R Studio (V.3.4.2) using a two-sided proportions test with the power of $80 \%$ and correct interpretation of the labels being $69 \%$ for standard labels and $91 \%$ for PCL.

\section{Survey instruments and administration procedure}

This randomised controlled trial was carried out using a parallel design structured interview approach. The structured interviews contained three parts and were administered face to face by one of the researchers (AS). The first part contained a demographic questionnaire to obtain participant characteristics: age (years), gender (male, female and other), level of education (primary, secondary, vocational, university), residential area (urban, rural), residency status (temporary, permanent resident/ citizen), annual income before tax (Australian Dollar, $\$ A$ ), private health insurance (yes, no), and language spoken by participants at home (English, other).

The second part contained two validated and widely used functional health literacy assessment tools including the Newest Vital Signs (NVS), ${ }^{26}$ and the Rapid Estimate of Adult Literacy in Medicine (REALM). ${ }^{27}$ The REALM explores word recognition and pronunciation skills, and the NVS evaluates prose, numeracy and document literacy skills. ${ }^{26} 27$ The REALM takes around $3 \mathrm{~min}$ to complete and involves reading aloud a list of 66 medical words arranged in increasing order of difficulty by its developers. ${ }^{27}$ The REALM score is calculated by awarding one score for each correctly pronounced word and no score for mispronounced or skipped words. A score of 59 or less indicates low health literacy, while a score of 60 or above indicates high health literacy. ${ }^{27}$ The NVS tool also takes around $3 \mathrm{~min}$ to complete and involves reading and understanding an ice-cream nutrition label and giving responses to a six-item questionnaire related to that label. ${ }^{26}$ The NVS score is calculated by awarding one score for each correct answer. A score of 4-6 indicates high health literacy, while a score of less than 4 indicates low health literacy. ${ }^{26}$

The third part of the survey contained two panels of three dispensing labels (standard labels or PCL). The standard labels were generated through Fred-Dispense software.$^{28}$ PCL were produced using Microsoft PowerPoint 2016 according to medication labelling guidance available in the literature on PCL. ${ }^{14}{ }^{15}$ The selection of medications was informed by previous literature. ${ }^{29-31}$ The appearance of the PCL was kept consistent with the current labelling practices used by many pharmacies in Australia, but the stylistic margins and headers were removed, and hypothetical or estimated whitespace available on the label was doubled $(70 \times 60 \mathrm{~mm})$ compared with the single standard label $(70 \times 30 \mathrm{~mm})$. Both standard and PCL version of labels used in this study are available in online supplemental file.

Participants were randomly assigned to receive either three standard labels or three PCL. The panel of labels contained dosing instructions that are frequently used and have been identified in the literature as possibly confusing for individuals. ${ }^{30}$ The allocated labels and a grid sheet printed on A3 size paper were provided to the participants. The grid sheet was arranged like a dosette box providing the times (columns) and days (rows) that the medicine would be taken. The participants were asked to comprehend instructions given in their assigned labels. The following questions were asked:

1 . What is the name of the medicine?

2. How would you take this medicine?

3. Indicate the dose that you would take throughout the day? (on the grid)

4. How long would you take the medicine for? (on the grid)

Participants were provided with placebo tablets (as a solid dosage form) and water (as a liquid dosage form). They were asked to interpret dispensing label instructions and put the required number of tables on the grid sheet. For doses of liquid solution, they were asked to measure the required $\mathrm{mL}$ of the liquid using an empty syringe. The interviewer (AS) captured a photograph of the grid sheet when participants put the tablets/syringe on the sheet or marked the grid sheet using a removable ink marker. Notes were made on a separate page by the interviewer.

\section{Data analysis}

Data were first entered into Microsoft Excel 2016 spreadsheet. Participant responses were converted into binary responses (correct or incorrect interpretation).

Participants were required to state the name of the medicine and identify the required number of tablets/ capsules or $\mathrm{mL}$ of liquid to be taken for each dose, the interval between doses and the overall treatment duration. Participant responses were considered correct if participants accurately indicated on the grid sheet both dose, interval and duration of treatment. Participants were also asked to identify the generic name of the medicine when they gave the name of the medicine, and at the end all participants were asked if they had anything else to state.

The dosing interval instructions in the PCL was explicit (morning (7AM-9 AM), lunchtime (11AM-1 PM), evening (4 PM-6 PM) and bedtime (9 PM-11 $\mathrm{PM}){ }^{22}$ therefore, participant responses were considered correct if their medication-taking time aligned with the prescribed time of the day in the label. A small variation in the dosing interval was considered acceptable for individuals who had a different sleep/wake, food intake or work pattern. Participants assigned with standard labels were given greater flexibility in terms of the 
medication-taking time and dosing interval. The key determinant was on whether the participant correctly understood the information presented and whether or not the participant's interpretation was likely to have clinical consequences. This decision was made to ensure any difference between PCL and standard labels was not overestimated. For example, correctly interpreting metformin twice daily (label 1), required the participant to identify metformin needed to be taken two time that day and suggest a minimum dosage interval of 6 hours or more. Two participants suggested they would take metformin in the morning and at lunch time because these were their only substantial meals. While this meant they might have taken the second dose of metformin within a 6-hour interval it was considered acceptable because the participant correctly interpreted the dose and metformin can be taken in this way. For flucloxacillin four times daily (label 2), a minimum interval of 3 hours or more was required between the first and second dose to be considered as a correct response. For prednisolone once daily (label 3), participants were asked to accurately measure the liquid dose with an empty syringe, and the response was considered correct if participants demonstrated to take $3 \mathrm{~mL}$ anytime in the day. For flucloxacillin (label 2) and prednisolone (label 3) participant responses were considered correct if they correctly described both the number of days and number of repeats. For metformin (label 1) participant responses were considered correct if they stated that no information was provided on how long they should take it or if they stated the number of repeats mentioned on the label.

The primary outcome measure of the study was the difference in the proportion of participants able to comprehend PCL compared with standard labels. A participant was judged to have correctly comprehended the medication label if they were able to (1) correctly state the name of the drug, (2) correctly describe the directions for the medicine provided on the label and (3) physically demonstrate the correct dosing of the medicine on the grid provided; this included identifying the correct dose, interval and duration of treatment as stated on the label.

Descriptive statistics were used to calculate frequencies, percentages, means and SD. $\chi^{2}$ and non-parametric tests were used to check group differences between participants assigned to control (standard) and intervention (PCL) groups. Test for two proportions was carried out using R Studio (V.3.4.2) to measure the difference in effect (effectiveness of PCL in improving the proportion of participants that correctly comprehend label information). This test was carried out to determine whether two different proportions or groups (PCL vs standard) differed significantly. Correct comprehension was also calculated for participants with low and high health literacy levels. Logistic regression analysis was also carried out that determines the relationship between the dependent variable and independent variables by estimating probabilities using a logistic regression equation. Logistic regression assessed the influence of health literacy, label format and other demographic variables (gender, education, residential area and private health insurance) on the proportion of participants correctly comprehending labels (dependant variable). Private health insurance was included to check whether there is any influence of affluence on comprehension of medication dosing.

\section{Patient and public involvement}

Participants were not involved in the study design, research questionnaire and outcomes measures development. Potential participants were invited to participate in this research and provided with participant information sheet and consent form. Participants were provided with an opportunity to ask any questions before agreeing to participate. Participants were informed that their participation in this research was voluntarily and that they could withdraw at any stage. Informed consent was obtained from those who agreed to participate in the study.

\section{RESULTS}

In total 155 consumers were approached and invited to participate in this study and 121 participated (figure 1); 60 were assigned to the control group (standard label) and 61 to the intervention group (PCL). Most participants were female $(57.9 \%)$, with a mean age of $43.8( \pm 13.3)$ years and income of $\$ A 70318.18( \pm 33965.95)$. The majority of participants were Australian permanent residents or citizens $(99.2 \%)$, more than $80 \%$ were residing in urban areas and almost half $(46.3 \%)$ had completed a university-level education.

The majority of participants were born in Australia (65.3\%), New Zealand (14.0\%), the UK (6.6\%) and Ireland $(2.5 \%)$. Almost 8 out of 10 participants spoke only English as a main language of communication at home. Close to $25 \%$ of the participants had private health insurance. Forty-five percent of participants had low scores on NVS, which assessed prose, numeracy, and document literacy, and $36.4 \%$ of participants had low scores on REALM, which assessed reading and pronunciation literacy. There were no significant differences between the control (standard) and intervention (PCL) groups in terms of participant demographics (table 1).

Participants who received PCL were significantly more likely to correctly comprehend and demonstrate dosing instructions. The proportion of participants who were able to accurately comprehend dosing instructions for all three labels was higher in the intervention group (PCL). The panel of standard labels was accurately comprehended by only $23.3 \%$ of participants, but the panel of PCL was accurately comprehended by $83.6 \%$ of participants $(\mathrm{p}<0.001)$ (table 2).

Participants accurately identified the name of the medicine for all the labels, and were able to state the label instructions, but they made errors when it came to demonstrating dose and dosing instructions for standard labels (table 3). Dose and dosing interval were 


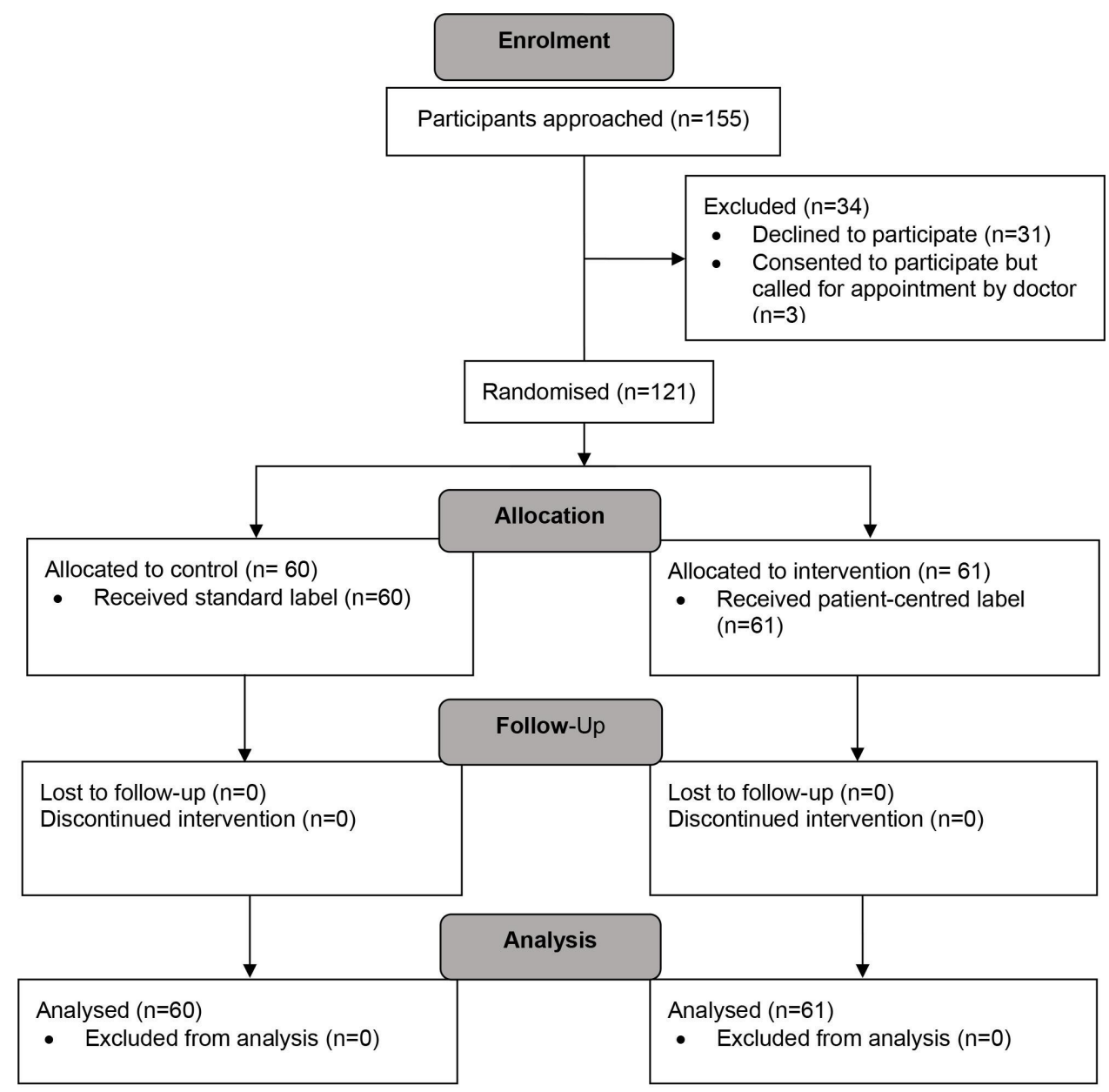

Figure 1 CONSORT diagram. CONSORT, Consolidated Standards of Reporting Trials.

accurately demonstrated by most of the participants in the PCL group. The duration of treatment was also accurately described by most of the participants assigned to the PCL group. The proportion of correctly identifying generic names of medicines and describing the information written on cautionary advisory labels was also higher in participants assigned to the PCL group.

The proportion of participants who accurately comprehended the label was higher in both low and high health literacy participants in the PCL group. However, accurate comprehension was greater in participants with low health literacy (table 4). Compared with the control group, a higher proportion of PCL group participants with low NVS score $(8.3 \%$ standard vs $85.7 \%$ PCL, $p<0.001)$ and low REALM score (10.5\% standard vs $96.0 \%$ PCL, $\mathrm{p}<0.001)$ were able to accurately comprehend the dosing information.

Logistic regression analysis showed that the odds of participants correctly comprehending PCL was higher than standard labels (OR 45.73, 95\% CI 12.61 to 165.88 , $\mathrm{p}<0.001)$ ). Participants with private health insurance had higher odds of correctly comprehending labels than those without private health insurance (OR 4.44, 95\% CI 1.24 to $15.87, \mathrm{p}=0.022)$ ). Participants with high NVS scores, which measures prose, numeracy and document literacy, also had higher odds of correctly comprehending labels than those with low NVS scores (OR 8.64, 95\% CI 1.59 to $46.86, \mathrm{p}=0.012$ )) (table 5). Participants with high REALM scores, which measures reading and pronunciation literacy, had OR of correctly comprehending labels close to zero $(0.10)$, which indicated that the effect size of this variable was really small.

\section{DISCUSSION}

Participants who received PCL were more likely to accurately comprehend key medicine labelling information compared with participants who received standard labels. The proportion of participants who accurately comprehended medicine labelling information was higher in both participants with high and low health literacy who received PCL.

The result of this study aligns with studies conducted elsewhere $^{151618}$ and provides evidence that PCL are more accurately comprehended by participants than standard labels. This study adds to the literature supporting implementation of PCL within the Australian context and internationally where dispensing labels are printed in the English language. It remains important to assess the effects of specific implementations of PCL in local contexts 
Table 1 Characteristics of recruited participants

\begin{tabular}{|c|c|c|c|c|c|}
\hline Variable & & Value & $\begin{array}{l}\text { Control group (Standard) } \\
\mathrm{n}=60\end{array}$ & $\begin{array}{l}\text { Intervention group (PCL) } \\
n=61\end{array}$ & $P$ value \\
\hline Age & Years & Mean (SD) & $41.73(12.13)$ & $45.85(14.15)$ & 0.090 \\
\hline Income* & AUD & Mean (SD) & 75098.21 (32386.78) & 64093.02 (35330.29) & 0.110 \\
\hline \multirow[t]{2}{*}{ Gender } & Male & N (\%) & $24(40.0)$ & $27(44.3)$ & 0.635 \\
\hline & Female & N (\%) & $36(60.0)$ & $34(55.7)$ & \\
\hline \multirow[t]{2}{*}{ Residency status } & PR holder or citizen & $\mathrm{N}(\%)$ & $60(100.0)$ & $60(98.4)$ & 0.319 \\
\hline & Temporary resident & N (\%) & $0(0.0)$ & $1(1.6)$ & \\
\hline \multirow[t]{2}{*}{ Area of residence } & Urban & N (\%) & $49(81.7)$ & $50(82.0)$ & 0.966 \\
\hline & Rural & $\mathrm{N}(\%)$ & $11(18.3)$ & $11(18.0)$ & \\
\hline \multirow[t]{3}{*}{ Education level } & Secondary or below & N (\%) & $20(33.3)$ & $18(29.5)$ & 0.579 \\
\hline & Trade or skilled & $\mathrm{N}(\%)$ & $11(18.3)$ & $16(26.2)$ & \\
\hline & University & $\mathrm{N}(\%)$ & $29(48.3)$ & $27(44.3)$ & \\
\hline \multirow[t]{7}{*}{ Country of birth } & Australia & $N(\%)$ & $37(61.7)$ & $42(68.9)$ & 0.243 \\
\hline & New Zealand & $\mathrm{N}(\%)$ & $7(11.7)$ & $10(16.4)$ & \\
\hline & UK & N (\%) & $3(5.0)$ & $5(8.2)$ & \\
\hline & Ireland & $\mathrm{N}(\%)$ & $3(5.0)$ & $0(0.0)$ & \\
\hline & South Africa & N (\%) & $2(3.3)$ & $0(0.0)$ & \\
\hline & India & N (\%) & $1(0.8)$ & $1(1.6)$ & \\
\hline & Other country* & $\mathrm{N}(\%)$ & $7(11.7)$ & $3(4.9)$ & \\
\hline \multirow{5}{*}{$\begin{array}{l}\text { Main spoken } \\
\text { language at home }\end{array}$} & English & N (\%) & $52(86.7)$ & $54(88.5)$ & 0.376 \\
\hline & English+Other† & $\mathrm{N}(\%)$ & $3(5.0)$ & $3(4.9)$ & \\
\hline & French & $\mathrm{N}(\%)$ & $0(0.0)$ & $2(3.3)$ & \\
\hline & Spanish & N (\%) & $2(3.3)$ & $0(0.0)$ & \\
\hline & Other language $\neq$ & N (\%) & $3(5.0)$ & 2 (3.3) & \\
\hline \multirow[t]{2}{*}{ Private insurance } & Yes & N (\%) & 17 (28.3) & $13(21.3)$ & 0.371 \\
\hline & No & N (\%) & $43(71.7)$ & $48(78.7)$ & \\
\hline \multirow{2}{*}{$\begin{array}{l}\text { Prose, numeracy, } \\
\text { and document } \\
\text { health literacy } \\
\text { (NVS) }\end{array}$} & $\operatorname{Low}(<4)$ & N (\%) & $24(40.0)$ & $28(45.9)$ & 0.512 \\
\hline & High (4-6) & $N(\%)$ & $36(60.0)$ & $33(54.1)$ & \\
\hline \multirow{2}{*}{$\begin{array}{l}\text { Reading and } \\
\text { pronunciation } \\
\text { health literacy } \\
\text { (REALM) }\end{array}$} & Low $(<60)$ & N (\%) & $19(31.7)$ & $25(41.0)$ & 0.287 \\
\hline & High (61-66) & N (\%) & $41(68.3)$ & $36(59.0)$ & \\
\hline
\end{tabular}

$\chi^{2}$ test and "non-parametric test was used to investigate group differences.

*Other countries: one participant each from Pakistan, Sweden, Argentina, Vietnam, Colombia, Fiji, France, Holland, Italy and Mexico.

†Other: one participant each speaking Greek, Malayalam, Italian, Gujrati, Thai and Spanish language at home. ¥Other language: one participant each speaking Urdu, Vietnamese or Unknown language (missing value) at home. AUD, Australian dollar; NVS, Newest Vital Signs; PCL, Patient-centred label; PR, Permanent Residence; REALM, Rapid Estimate of Adult Literacy in Medicine.

because not all implementations appear to be effective. For example, in a US study, the inclusion of graphic aid or pictograms on labels did not provide any benefit to those with limited health literacy. ${ }^{16}$ A recent randomised control trial conducted in Ireland also reported that PCL did not statistically improve comprehension of directions in participants (mean score 9.28 vs $8.81, \mathrm{p}=0.135$ ) ${ }^{32}$ The Irish study further reported that PCL did not provide any additional benefits for participants with low health literacy (Mean score 8.56 vs 9.06, $p=0.514) .{ }^{32}$ One notable limitation of the Irish study is that it did not recruit the desired sample size, which could show statistical power difference between the intervention and control group. The expected sample size calculated using power sampling formula was 100 participants, but the study could only recruit 76 participants. This might have seriously impacted the results of the Irish study. 
Table 2 Comparison of the number and \% of participants correctly comprehending the labels for the control (standard label) and intervention (patient-centred label (PCL)) groups

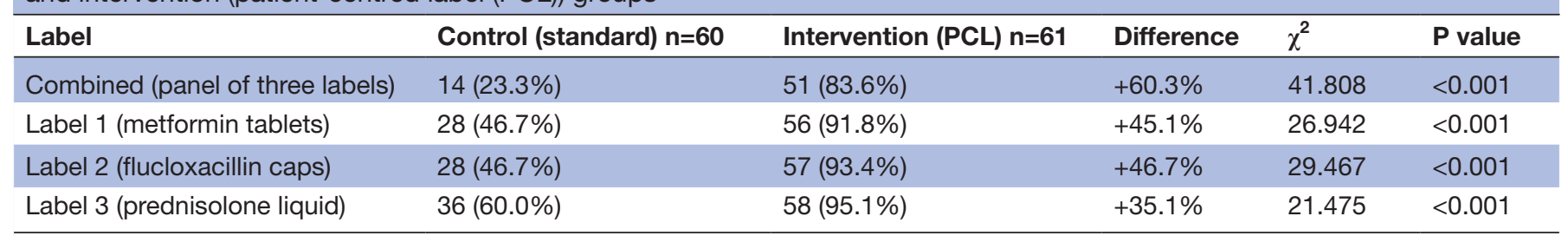

Comprehension of labels was considered correct if participants gave correct answers to all questions, excluding the identification of generic name and description of cautionary label instructions; $\chi^{2}$, with $1 \mathrm{df} ; \mathrm{p}$ values were calculated using two proportion test (two sided) in R software.

This study, like others, ${ }^{6} 16$ reports that PCL are more accurately comprehended than standard labels by all participants irrespective of their health literacy levels. Pharmacists often use the 'teach-back' technique in clinical practice to confirm patients' understanding of the provided medicine-related information..$^{33}$ This technique may not truly represent the actual medicationtaking behaviour of patients or medication adherence, particularly for those with lower health literacy, but may identify patients at risk of misunderstanding information and misusing medications. Davis et al reported that when patients with lower health literacy were asked to state dispensing label instructions provided on standard labels and demonstrate how they would take the medicine, about $70 \%$ of patients stated instructions accurately, but only $34 \%$ could demonstrate the actual required number of tablets. ${ }^{6}$ This has also been observed in our study. For example, almost all of the participants accurately stated standard label instructions, but only two-thirds (slightly above 60\%) correctly demonstrated dose and dosing interval. When compared with standard labels, participants provided with PCL were better at demonstrating dosing instructions. This indicates that implementing PCL may reduce the burden on pharmacists, it may reduce their need to use the tech-back technique and may help overcome the risk of medication misuse associated with standard labels.

This study reports another interesting finding that participants assigned to the PCL group were better at stating cautionary label instructions despite being provided with the same cautionary labels. Although participants were not asked to state cautionary label instructions, many stated those instructions when they were asked how they would take their medicines. This might indicate that participants provided with standard labels consume a lot of time in understanding information, which indirectly affects their attention to further details. Lack of attention to cautionary labels is not a new phenomenon and has been reported by other studies. ${ }^{36}$ The lack of attention of participants to these labels could be due to their perceived lack of importance of the instructions provided by cautionary labels. ${ }^{34}{ }^{35}$ However, the actual reason behind the lack of attention to advisory labels remains unknown and presents a potential area of further research. Unveiling this phenomenon may help healthcare providers to understand the actual problem and to highlight the importance of cautionary label instructions to patients to optimise their medicationtaking behaviours.

Table 3 Number and percentage of participants who correctly interpreted and demonstrated the specific scoring criteria for each label $(\mathrm{N}=121)$

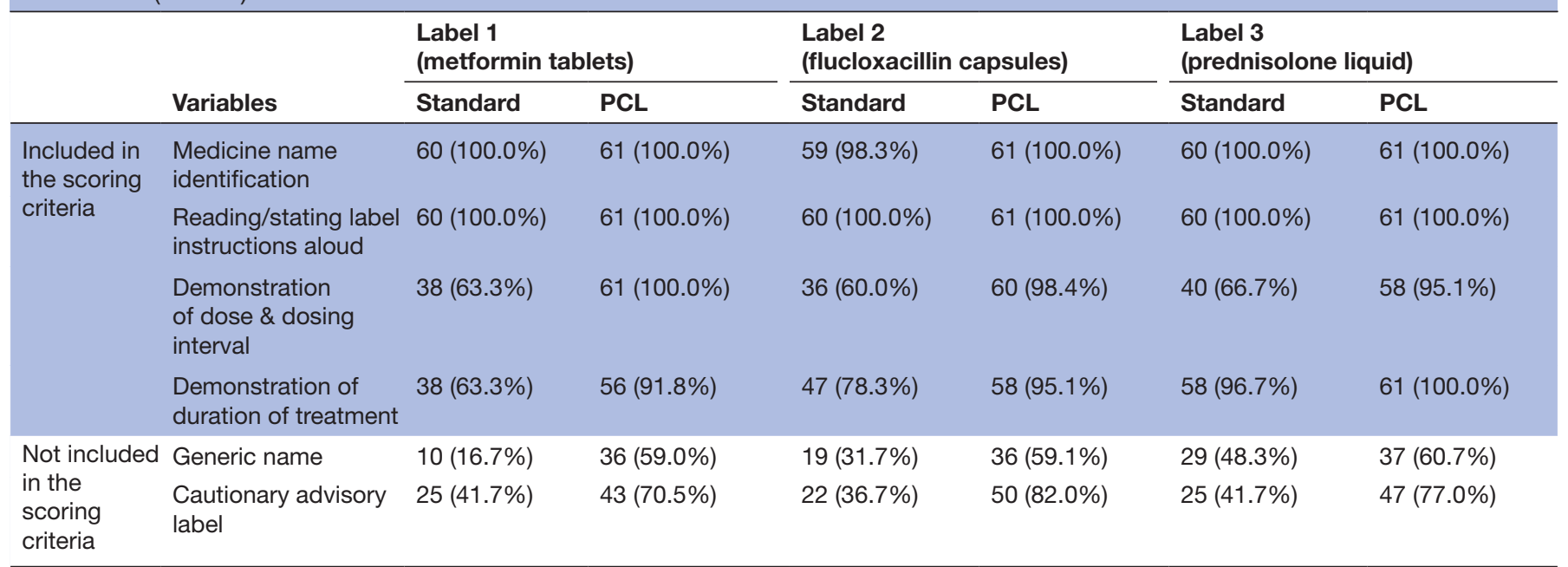

PCL, patient-centred labels. 
Table 4 Comparison of the percentage of participants who correctly comprehended standard labels and patient-centred labels (PCL) for those with high and low health literacy $(\mathrm{HL})$ according to their answers to the Newest Vital Signs (NVS) and Rapid Estimate of Adult Literacy in Medicine (REALM) questionnaires

\begin{tabular}{|c|c|c|c|c|c|c|}
\hline HL scale & HL level (scores) & Control (standard) & Intervention (PCL) & Percentage difference & $\chi^{2}$ & $P$ value \\
\hline \multirow[t]{2}{*}{ NVS } & Low $(<4)$ & $8.3 \%(2 / 24)$ & $85.7 \%(24 / 28)$ & +77.4 & 27.935 & $<0.001$ \\
\hline & High (4-6) & $33.3 \%(12 / 36)$ & $81.8 \%(27 / 33)$ & +48.5 & 14.556 & $<0.001$ \\
\hline REALM & High (61-66) & $29.3 \%(12 / 41)$ & $75.0 \%(27 / 36)$ & +45.7 & 14.261 & $<0.001$ \\
\hline
\end{tabular}

$\chi^{2}$ : with $1 \mathrm{df} ; \mathrm{p}$ values were calculated using two proportions test (two sided).

The regression analysis revealed that participants assigned to the PCL group and those with higher REALM and NVS score had higher odds of correctly comprehending dosing instructions. These findings are also in line with previous literature. ${ }^{16}$ Although PCL improved comprehension in participants with high health literacy, this effect was comparatively less than participants with lower health literacy. This might be due to the limitation of the functional health literacy assessment tools used in this study. Scoring high on REALM scale may not directly equate with participants being labelled as someone with higher health literacy as it involves only reading aloud medical terminologies. Similarly, scoring lower on NVS scale does not always mean that participants have lower health literacy. Sometimes patients might not be familiar with the NVS tool and find it difficult to respond to NVS items. In fact, both REALM and NVS have been criticised because of these limitations. ${ }^{36} 37$ Both NVS and REALM have been commonly used in pharmacy practice and PCL research. ${ }^{15162938}$ Therefore, we used those tools to allow comparison with previous research. The regression analysis also revealed a unique finding that participants with private health insurance had higher odds of correctly comprehending dosing instructions. This indicates a mixed effect of affluence and health literacy on comprehension, because private health insurance is regarded as a proxy measure of socioeconomic status and health literacy, ${ }^{39} 40$ and those with private health insurance are reported to be better in health literacy domains of actively managing health and understanding health information. $^{39}$

The findings of this study support the implementation of PCL in Australia as recommended by the ACSQHC. ${ }^{19} 22$ While the implementation of the full suite of PCL recommendations would require actions from multiple stakeholders, the implementation of PCL used in this study can be readily implemented in contemporary pharmacy practice. The use of 'extra white spacing', which meet the dimensions of PCL used in this study, could be implemented without requiring pharmacies to purchase new printers or hardware devices. The second aspect of the intervention (PCL instructions) is also implementable as

Table 5 Predictors for correctly comprehending labels, calculated using logistic regression

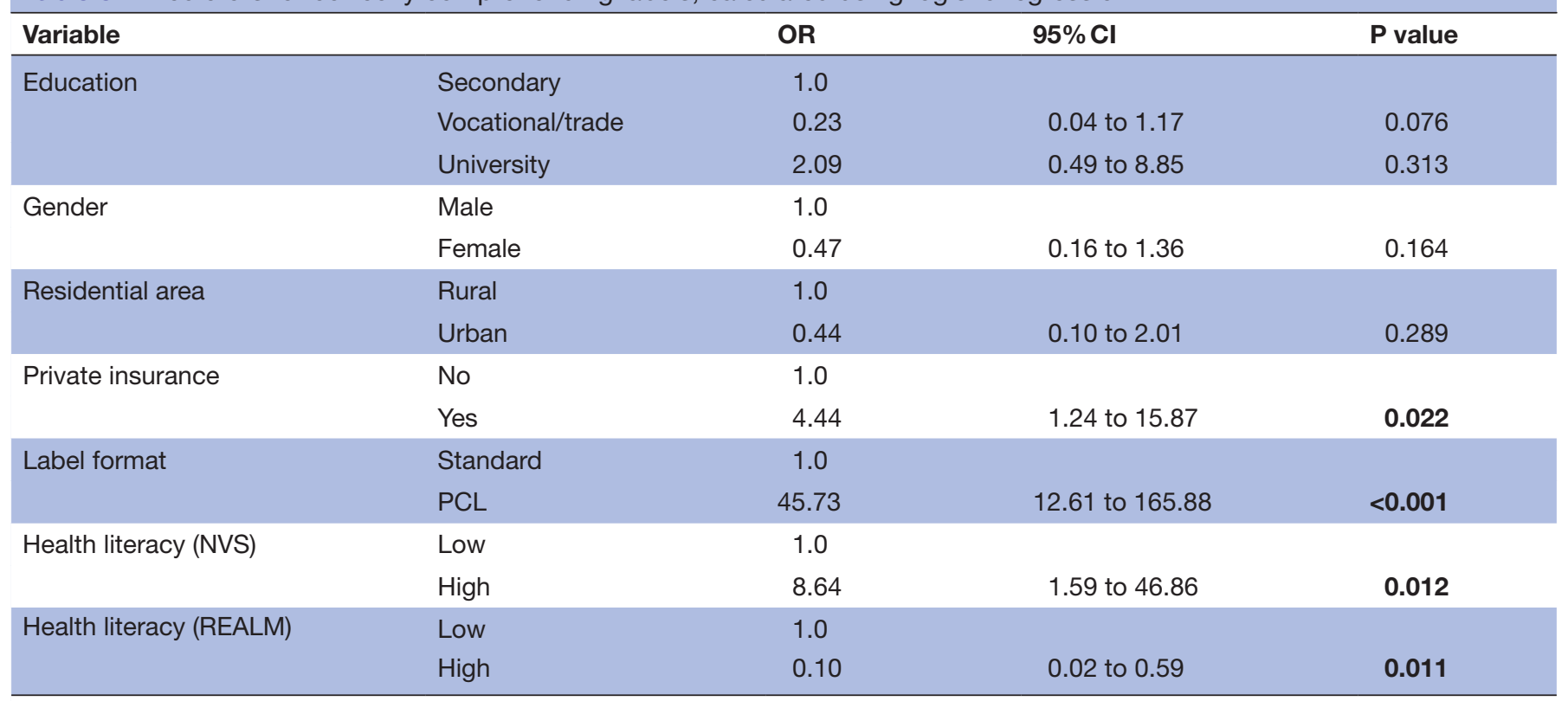

Bold $p$ values are significant $(\leq 0.05)$,

NVS, Newest Vital Signs; PCL, patient-centred label; REALM, Rapid Estimate of Adult Literacy in Medicine. 
pharmacies normally use short-hand codes to produce medication dosing instructions for dispensing labels. These codes are either prepopulated by the software vendors or user-defined by the pharmacies. ${ }^{22}$ Both these aspects could be implemented easily if dispensing software vendors make slight changes to the software systems.

Implementing PCL in Australia could be further aided by addressing some policy, regulatory and resource-related challenges. This would include clarifying existing policies so that they provide clear guidance on appropriate labelling practices. For example, the peak professional body for pharmacists in Australia (the Pharmaceutical Society of Australia) could clarify professional standards concerning dispensing labels and explicitly define what encompasses 'ensures that the font size of labelling is appropriate to the recipient (where possible) ${ }^{41}$ Changes are also required in the dispensing label legislative requirements that guide on the dimensions of labels and the information that needs to be printed on the label. ${ }^{21} 22$

This study has some important strengths and limitations that need to be considered by the readers. First, although we approached both non-English speaking (CALD) and English speaking (non-CALD) consumers, the majority of recruited participants were from non-CALD background. Unfortunately, many CALD consumers, perceived by the interviewer to be of East-Asian origin, declined the invitation to participate in the study. The exact reason is not known, but their hesitation could be due to their lack of language proficiency or discomfort with the interviewer who represented a different cultural background to their own. Further research is needed to test the effectiveness of PCL in both prominent and emerging CALD communities. Second, the majority of participants were from Englishspeaking backgrounds therefore further work is needed in specific vulnerable groups including Aboriginal and Torres Strait Islanders. Third, some potential participants declined to participate because they were not offered any compensation in return for their time investment. Offering compensation may improve the response rate of individuals in future studies. Two approaches were used for data collection: (1) pharmacy staff at the counter directed patients towards the researcher and explained study purpose briefly, and (2) the researcher approached individuals directly, introduced himself, and briefly introduced study. The first approach did not work well due to two reasons, it disrupted the workflow of the pharmacy putting extra task burden on pharmacy staff, and it made both the participants and the pharmacy staff uncomfortable. Compared with the first approach, the second approach was more effective. Therefore, researchers conducting future work are advised to approach participants directly to avoid extra burden on staff. Fourth, this study did not investigate the actual medication-taking practices of patients and medication adherence. Previous studies have investigated the impact of PCL on medication adherence and the available evidence on the impact on adherence is mixed. ${ }^{152-45}$ Future studies may further explore the impact of the PCL on medication adherence, however this was not the aim of this study.
Lastly, even though participants were randomised on entry into this study, the intervention group mean age was 4 years greater than the control group.

In terms of strengths, the use of power sampling to calculate desired sample size, and 1:1 simple randomisation, makes this study robust and generalisable. Additionally, participants recruited in this study were from diverse socioeconomic backgrounds, with different education, income and health literacy levels, and no significant group differences were observed between the control and intervention group. Third, the interventions developed and used this study retain the appearance and formatting of current labels, an intentional design to allow changes to PCL to be readily implemented in Australian pharmacy practice.

\section{CONCLUSIONS}

Dispensing labels are often the last information resource for patients before they actually take their medicine. Any misinterpretation of instruction may lead to compromised health outcomes or possible harm due to misuse of medicine. This study provides further evidence that the use of PCL improve comprehension of key directions for medication use in patients. Implementing the PCL format in Australian pharmacy practice may reduce the risk of misinterpreting instruction by patients and improve the quality use of medicines.

\section{Twitter Adam La Caze @adamlacaze}

Acknowledgements We would like to thank Professor lan Coombes (Director Pharmacy, RBWH), Danielle Silvestro (Cancer Care Pharmacist, RBWH) and other pharmacy staff at RBWH for providing support throughout the data collection process. We would like to acknowledge that AS was supported by the University of Queensland Research Training Program (RTP) scholarship for his doctoral research.

Contributors AS, KS and ALC conceived and designed the study; AS designed dispensing labels and data collection forms with help from KS and ALC; AS prepared the protocol and ethics and governance application with help from KS and ALC; GW helped with the site specific ethics and governance application and supervised the data collection; AS recruited participants, collected data and carried out the statistical analysis with help from ALC; AS wrote the manuscript that was critically reviewed by ALC and KS; GW also provided feedback on the manuscript; and all authors read and approved the manuscript. All authors take responsibility for the work, had access to the data, and controlled the decision to publish.

Funding The authors have not declared a specific grant for this research from any funding agency in the public, commercial or not-for-profit sectors.

Competing interests None declared.

Patient consent for publication Not applicable.

Ethics approval This study was approved by the Royal Brisbane Women's Hospital (RBWH) Human Research Ethics Committee (Approval: LNR/2019/QRBW/55782) and also from the Human Research Ethics Committee (HABS Faculty LNR SubCommittee) of The University of Queensland (Approval: 2019002315/LNR/2019/ QRBW/55782). Informed consent was obtained from participants after providing them detailed information about the project both verbally and with the help of the participant information sheet.

Provenance and peer review Not commissioned; externally peer reviewed.

Data availability statement Data are available on reasonable request. Data are available upon reasonable request. Study protocol and non-identifiable participant data that underlie the results reported in this article have been stored securely on the University of Queensland's Research Data Manager (RDM) system. This data and study protocol will be available immediately following publication for 15 years. Data will be shared with researchers who provide a methodologically sound proposal approved by an independent ethics review committee. Proposals should 
be directed to AS (a.saleem@uqconnect.edu.au) or ALC (a.lacaze@uq.edu.au).To gain access, data requesters will need to sign a data access agreement.

Supplemental material This content has been supplied by the author(s). It has not been vetted by BMJ Publishing Group Limited (BMJ) and may not have been peer-reviewed. Any opinions or recommendations discussed are solely those of the author(s) and are not endorsed by BMJ. BMJ disclaims all liability and responsibility arising from any reliance placed on the content. Where the content includes any translated material, BMJ does not warrant the accuracy and reliability of the translations (including but not limited to local regulations, clinical guidelines, terminology, drug names and drug dosages), and is not responsible for any error and/or omissions arising from translation and adaptation or otherwise.

Open access This is an open access article distributed in accordance with the Creative Commons Attribution Non Commercial (CC BY-NC 4.0) license, which permits others to distribute, remix, adapt, build upon this work non-commercially, and license their derivative works on different terms, provided the original work is properly cited, appropriate credit is given, any changes made indicated, and the use is non-commercial. See: http://creativecommons.org/licenses/by-nc/4.0/.

\section{ORCID iD}

Ahsan Saleem http://orcid.org/0000-0003-1710-3578

\section{REFERENCES}

1 Tai B-WB, Bae YH, LaRue CE, et al. Putting words into action: a simple focused education improves prescription label comprehension and functional health literacy. J Am Pharm Assoc 2016;56:145-52.

2 Wolf MS, Davis TC, Shrank W, et al. To err is human: patient misinterpretations of prescription drug label instructions. Patient Educ Couns 2007;67:293-300.

3 Davis TC, Wolf MS, Bass PF, et al. Low literacy impairs comprehension of prescription drug warning labels. J Gen Intern Med 2006;21:847-51.

4 Patel MJ, Khan MS, Ali F, et al. Patients' insight of interpreting prescriptions and drug labels--a cross sectional study. PLoS One 2014;8:e65019.

5 Leyva M, Sharif I, Ozuah PO. Health literacy among spanishspeaking latino parents with limited english proficiency. Ambul Pediatr 2005;5:56-9

6 Davis TC, Wolf MS, Bass PF, et al. Literacy and misunderstanding prescription drug labels. Ann Intern Med 2006;145:887-94.

7 Koster ES, Blom L, Winters NA, et al. Interpretation of drug label instructions: a study among four immigrants groups in the Netherlands. Int J Clin Pharm 2014;36:274-81.

8 Shrank W, Avorn J, Rolon C, et al. Effect of content and format of prescription drug labels on readability, understanding, and medication use: a systematic review. Ann Pharmacother 2007;41:783-801.

9 Nutbeam D. Defining and measuring health literacy: what can we learn from literacy studies? Int J Public Health 2009;54:303-5.

10 Koster ES, Philbert D, Bouvy ML. Health literacy among pharmacy visitors in the Netherlands. Pharmacoepidemiol Drug Saf 2015;24:716-21.

11 Wolf MS, Shekelle P, Choudhry NK, et al. Variability in pharmacy interpretations of physician prescriptions. Med Care 2009;47:370-3.

12 Borgsteede SD, Heringa M. Nature and frequency of dosing instructions on prescription labels in primary care. Pharmacoepidemiol Drug Saf 2019;28:1060-6.

13 Leat SJ, Ahrens K, Krishnamoorthy A, et al. The legibility of prescription medication labelling in Canada: moving from pharmacycentred to patient-centred labels. Can Pharm J 2014;147:179-87.

14 Institute of Medicine. Standardizing medication labels: confusing patients less: workshop summary. Washington, DC: The National Academies Press, 2008.

15 Wolf MS, Davis TC, Curtis LM, et al. A patient-centered prescription drug label to promote appropriate medication use and adherence. $J$ Gen Intern Med 2016;31:1482-9.

16 Wolf MS, Davis TC, Curtis LM, et al. Effect of standardized, patientcentered label Instructions to improve comprehension of prescription drug use. Med Care 2011;49:96-100.

17 Sahm LJ, Wolf MS, Curtis LM, et al. What's in a label? An exploratory study of patient-centered drug instructions. Eur J Clin Pharmacol 2012;68:777-82.

18 Bailey SC, Sarkar U, Chen $\mathrm{AH}$, et al. Evaluation of language concordant, patient-centered drug label instructions. J Gen Intern Med 2012;27:1707-13.
19 Australian Commission on Safety and Quality in Health Care. National standard for labelling dispensed medicines. Sydney, NSW: ACSQHC, 2021.

20 StirlingFildes. Pharmacy dispensing labels. Available: https://www. stirlingfildes.com.au/PharmacyDispensingLabels [Accessed $11 \mathrm{Sep}$ 2021].

21 Therapeutic Good Administration. TGA medicine labelling and packaging review. Department of Health, Australian Government, 2012. https://www.tga.gov.au/book/dispensing-label-space

22 Australian Commission on Safety and Quality in Health Care. Improving the safety and quality of pharmacy dispensing labels. In: National round table report ACSQHC. Sydney: NSW Clinical Excellence Commission, 2014.

23 Tong V, Aslani P, Raynor DK, et al. Developing and user testing new pharmacy label formats -A study to inform labelling standards. Health Expect 2021;24:1125-36.

24 Guo M, Carter SR, Lau E, et al. User testing to examine patient understanding of pharmacy generated medication labels. Patient Educ Couns 2020;103:2290-6.

25 Aslani P, Tong V. Developing standards for labelling dispensed medicines. Sydney, NSW: The University of Sydney, 2019.

26 Weiss BD, Mays MZ, Martz W, et al. Quick assessment of literacy in primary care: the newest vital sign. Ann Fam Med 2005;3:514-22.

27 Davis TC, Long SW, Jackson RH, et al. Rapid estimate of adult literacy in medicine: a shortened screening instrument. Fam Med 1993;25:391-5.

28 Fred Dispense. Australia: Fred it group, 2018. Available: https://www. fred.com.au/what-we-do/dispensary/fred-dispense/[Accessed 19 Nov 2018].

29 Davis TC, Federman AD, Bass PF, et al. Improving patient understanding of prescription drug label instructions. J Gen Intern Med 2009;24:57-62.

30 La Caze A. Safer dispensing labels for prescription medicines. Aust Prescr 2018;41:46-9.

31 Robinson J, McKenzie C, MacLeod D. Paediatric dosing errors with oral prednisolone mixture. Aust Prescr 2016;39:176-76.

32 McManus E, McCarthy S, Carson R, et al. Impact of a universal medication schedule on rationalising and understanding of medication; a randomised controlled trial. Res Social Adm Pharm 2018;14:831-8.

33 Schillinger D, Piette J, Grumbach K, et al. Closing the loop: physician communication with diabetic patients who have low health literacy. Arch Intern Med 2003;163:83-90.

34 Berkman ND, Sheridan SL, Donahue KE, et al. Low health literacy and health outcomes: an updated systematic review. Ann Intern Med 2011;155:97-107.

35 Kairuz TE, Bellamy KM, Lord E, et al. Health literacy among consumers in community pharmacy: perceptions of pharmacy staff. Health Expect 2015;18:1041-51.

36 Collins SA, Currie LM, Bakken S, et al. Health literacy screening instruments for eHealth applications: a systematic review. J Biomed Inform 2012;45:598-607.

37 Ibrahim SY, Reid F, Shaw A, et al. Validation of a health literacy screening tool (realm) in a UK population with coronary heart disease. J Public Health 2008;30:449-55.

38 Aslani P, Tong V. Assessing the impact of label format on people's ability to interpret and apply prescription medicine information. Sydney, NSW: The University of Sydney, 2020.

39 Beauchamp A, Buchbinder R, Dodson S, et al. Distribution of health literacy strengths and weaknesses across socio-demographic groups: a cross-sectional survey using the health literacy questionnaire (HLQ). BMC Public Health 2015;15:678.

40 Sentell T. Implications for reform: survey of California adults suggests low health literacy predicts likelihood of being uninsured. Health Aff 2012;31:1039-48.

41 Pharmaceutical Society of Australia (PSA). Professional practice standards. Deakin West, ACT, 2017.

42 Dowse R, Ehlers M. Medicine labels incorporating pictograms: do they influence understanding and adherence? Patient Educ Couns 2005;58:63-70.

43 Kripalani S, Schmotzer B, Jacobson TA. Improving medication adherence through Graphically enhanced interventions in coronary heart disease (IMAGE-CHD): a randomized controlled trial. J Gen Intern Med 2012;27:1609-17.

44 Bailey SC, Navaratnam P, Black H, et al. Advancing best practices for prescription drug labeling. Ann Pharmacother 2015;49:1222-36.

45 Murray MD, Young J, Hoke S, et al. Pharmacist intervention to improve medication adherence in heart failure: a randomized trial. Ann Intern Med 2007;146:714-25. 